\title{
On Planar Mixed Hypergraphs
}

\author{
Zdeněk Dvořák* \\ Department of Applied Mathematics \\ Charles University \\ Malostranské náměstí 25 \\ 11800 Prague, Czech Republic \\ rakdver@kam.ms.mff.cuni.cz
}

\author{
Daniel Král’† \\ Department of Applied Mathematics and \\ Institute for Theoretical Computer Science ${ }^{\ddagger}$ \\ Charles University \\ Malostranské náměstí 25 \\ 11800 Prague, Czech Republic \\ kral@kam.ms.mff.cuni.cz
}

Submitted: July 16, 2001; Accepted: October 12, 2001.

MR Subject classifications: Primary 05C15, Secondary 05C85, 68R10

Keywords: coloring of hypergraphs, planar graphs and hypergraphs, mixed hypergraphs, algorithms for coloring

\begin{abstract}
A mixed hypergraph $H$ is a triple $(V, \mathcal{C}, \mathcal{D})$ where $V$ is its vertex set and $\mathcal{C}$ and $\mathcal{D}$ are families of subsets of $V, \mathcal{C}$-edges and $\mathcal{D}$-edges. A mixed hypergraph is a bihypergraph iff $\mathcal{C}=\mathcal{D}$. A hypergraph is planar if its bipartite incidence graph is planar. A vertex coloring of $H$ is proper if each $\mathcal{C}$-edge contains two vertices with the same color and each $\mathcal{D}$-edge contains two vertices with different colors. The set of all $k$ 's for which there exists a proper coloring using exactly $k$ colors is the feasible set of $H$; the feasible set is called gap-free if it is an interval. The minimum (maximum) number of the feasible set is called a lower (upper) chromatic number.

We prove that the feasible set of any planar mixed hypergraph without edges of size two and with an edge of size at least four is gap-free. We further prove that a planar mixed hypergraph with at most two $\mathcal{D}$-edges of size two is two-colorable.

We describe a polynomial-time algorithm to decide whether the lower chromatic number of a planar mixed hypergraph equals two. We prove that it is NP-complete to find the upper chromatic number of a mixed hypergraph even for 3-uniform planar bihypergraphs. In order to prove the latter statement, we prove that it is NP-complete to determine whether a planar 3-regular bridgeless graph contains a 2 -factor with at least a given number of components.
\end{abstract}

${ }^{*}$ The author acknowledges partial support by GAČR 201/1999/0242 and GAUK 158/1999.

†The author acknowledges partial support by GAČR 201/1999/0242, GAUK 158/1999 and KONTAKT 338/99.

${ }^{\ddagger}$ Institute for Theoretical Computer Science is supported by Ministry of Education of Czech Republic as project LN00A056. 


\section{Introduction}

Planar graphs attract lots of attention of computer scientists. We consider a generalization of planar hypergraphs, called planar mixed hypergraphs, in this paper. A hypergraph is a pair $(V, \mathcal{E})$ where $\mathcal{E}$ is a family of subsets of $V$ of size at least 2 ; the members of $V$ are called vertices and the members of $\mathcal{E}$ are called edges. Hypergraphs are widely studied combinatorial objects, see [1]. A hypergraph is planar if its bipartite incidence graph is planar. A hypergraph is $k$-uniform if the size of each of its edges is exactly $k$. Mixed hypergraphs were introduced in [22], planar hypergraphs were introduced in [24] and the concept of planar mixed hypergraphs was first studied in [15] and further investigated in $[10]$.

A mixed hypergraph $H$ is a triple $(V, \mathcal{C}, \mathcal{D})$ where $\mathcal{C}$ and $\mathcal{D}$ are families of subsets of $V$; the members of $\mathcal{C}$ are called $\mathcal{C}$-edges and the members of $\mathcal{D}$ are called $\mathcal{D}$-edges. A proper $k$-coloring $c$ of $H$ is a mapping $c: V \rightarrow\{1, \ldots, k\}$ such that there are two vertices with $\mathcal{D}$ ifferent colors in each $\mathcal{D}$-edge and there are two vertices with a $\mathcal{C}$ ommon color in each $\mathcal{C}$-edge. The coloring $c$ is a strict $k$-coloring if it uses all $k$ colors. The mixed hypergraph is colorable iff it has a proper coloring. The concept of mixed hypergraphs can find its applications in different areas: coloring block designs, list-coloring of graphs and others (see $[2,3,4,16,18,19,20])$.

We will understand mixed hypergraphs as quadruples $(V, \mathcal{B}, \mathcal{C}, \mathcal{D})$ where the families $\mathcal{B}, \mathcal{C}$ and $\mathcal{D}$ of subsets of $V$ are mutually disjoint; the edges of $\mathcal{B}$ are $\mathcal{B}$ oth $\mathcal{C}$-edges and $\mathcal{D}$-edges of the mixed hypergraph. A mixed hypergraph $H$ is planar iff its bipartite incidence graph $B(H)$ is planar: The vertices of $B(H)$ are both vertices and edges of $H$, i.e. $V \cup \mathcal{B} \cup \mathcal{C} \cup \mathcal{D}$; a vertex $v \in V$ of $H$ and an edge $e \in \mathcal{B} \cup \mathcal{C} \cup \mathcal{D}$ are joined by an edge in $B(H)$ iff $v \in e$. A mixed hypergraph is a bihypergraph if $\mathcal{C}=\mathcal{D}=\emptyset$.

The feasible set $\mathcal{F}(H)$ of a mixed hypergraph $H$ is the set of all $k$ 's such that there exists a strict $k$-coloring of $H$. The (lower) chromatic number $\chi(H)$ of $H$ is the minimum number in $\mathcal{F}(H)$ and the upper chromatic number $\bar{\chi}(H)$ of $H$ is the maximum number in $\mathcal{F}(H)$. The feasible set of $H$ is gap-free (unbroken) iff $\mathcal{F}(H)=[\chi(H), \bar{\chi}(H)]$; we use $[a, b]$ for the set of all the integers between $a$ and $b$ (inclusively). The feasible set of $H$ has a gap at a number $k$ if $k \notin \mathcal{F}(H)$ and there exist $k_{1}<k<k_{2}$ such that $k_{1}, k_{2} \in \mathcal{F}(H)$. If the feasible set of $H$ contains a gap, we say it is broken. An example of a mixed hypergraph with a broken feasible set was first given in [9]. The question whether there exists also a planar mixed hypergraph with a broken feasible set was raised in [15] and the first example of a planar mixed hypergraph with a broken feasible set was obtained in [10].

Some questions about coloring properties of planar mixed hypergraphs were raised in [23] (problem 8, p. 43); some of their properties were described and some other open problems related to them were raised in [15]. Those problems include: Is it NP-hard to determine the upper chromatic number of a strongly-embeddable 3-uniform planar bihypergraph? (question 4) Are there planar mixed (bi)hypergraphs (without edges of size two) with a broken feasible set? (question 5) We deal with these questions in this paper.

The four color theorem for planar graphs and for planar mixed hypergraphs (a col- 
orable mixed planar hypergraph can be properly colored by at most four colors) are equivalent due to Kobler and Kündgen (see [10]). Moreover, Kobler and Kündgen proved in [10] that if the upper chromatic number of a mixed planar hypergraph $H$ is at least 4, then its feasible set $\mathcal{F}(H)$ contains the interval $[4, \bar{\chi}(H)]$. Thus the feasible set of a mixed planar hypergraph can contain a gap only at the number 3 (since if its lower chromatic number is one, then it contains only $\mathcal{C}$-edges and its feasible set is gap-free).

We summarize some basic properties of (planar) mixed hypergraphs in Section 2. We recall the concept of reductions on mixed hypergraphs previously used in $[11,12,13,22$, 23]. We further study in Section 2 an alternative definition of planar mixed hypergraphs - similar properties of planar mixed hypergraphs were already used in [10, 15].

We address properties of feasible sets and existence of strict colorings of planar mixed hypergraphs in Section 3. We prove that any planar mixed hypergraph with at most two $\mathcal{D}$-edges of size two is two-colorable (Theorem 1). This generalizes both the result of Kündgen et al. (see [15]) that any planar mixed hypergraph without edges of size two is two-colorable and the result of Burshtein and Kostochka (see [24]) that any planar mixed hypergraph with $\mathcal{B}=\mathcal{C}=\emptyset$ and with at most one $\mathcal{D}$-edge of size two is two-colorable. It was proved in [15] that the feasible set of any strongly-embeddable planar three-uniform mixed bihypergraph (i.e. that which contains only $\mathcal{B}$-edges and all its edges have size three) is gap-free. We generalize this result in particular to any strongly-embeddable (for the definition of a strong embedding see Section 2) planar mixed bihypergraph (Corollary 1). We prove that the feasible set of any planar mixed hypergraph without edges of size two and with an edge of size at least four is gap-free (Theorem 2). This gives a partial answer to the question 5 from [15]: There is no strongly-embeddable planar mixed bihypergraph with a broken feasible set and if there is a planar mixed hypergraph without edges of size two with a broken feasible set, then it is 3-uniform.

We address complexity questions dealing with colorings of planar mixed hypergraphs in Section 4 . We prove that there is a polynomial-time algorithm for determining whether the lower chromatic number of a planar mixed hypergraph equals 2 in Theorem 3 ; determining whether the lower chromatic number of a planar mixed hypergraph equals 1 is trivial, since all planar mixed hypergraphs with the lower chromatic number equal to 1 contain only $\mathcal{C}$-edges and determining whether the lower chromatic number of a planar mixed hypergraph is 3 is NP-complete even for planar graphs (see [6]) - for a summary of the results see Corollary 2. The lower chromatic number cannot exceed 4 due to the four color theorem. We answer the question 4 raised in [15] by proving that it is NP-complete to determine the upper chromatic number of a given planar mixed hypergraph even for strongly-embeddable 3-uniform planar bihypergraphs such that their strong embeddings do not contain parallel edges (Theorem 4). Proper colorings of a strongly-embeddable 3uniform planar bihypergraph $H$ correspond to the 2-factors of the dual graph of a strong embedding of $H$. The upper chromatic number of $H$ is equal to the maximum number of components of a 2-factor of the dual graph increased by one (see Lemma 4). Thus the corresponding complexity question can be rephrased as "Does a 3-regular bridgeless planar graph (with some additional properties) have a 2-factor with at least a given number of components?" - see Lemma 3 for the proof of NP-completeness of this problem; the 
NP-completeness of this problem is of its own theoretical interest. The NP-completeness of a similar problem of finding a 2-factor with exactly (or at most) one (i.e. a Hamiltonian cycle) for cubic planar graphs was established in [7].

\section{Basic Properties of Planar Mixed Hypergraphs}

We are interested in properties of feasible sets of planar mixed hypergraphs. The presence of $\mathcal{B}$-edges of size two implies noncolorability of a mixed hypergraph. We restrict our attention only to mixed hypergraphs without such edges (the first condition below). A mixed hypergraph is reduced if the following conditions hold (cf. [11, 12, 13, 22, 23]):

- The size of any $\mathcal{B}$-edge or $\mathcal{C}$-edge is at least three.

- There are no two edges $e \in \mathcal{B} \cup \mathcal{C}$ and $e^{\prime} \in \mathcal{B} \cup \mathcal{C}$ such that $e \subset e^{\prime}$.

- There are no two edges $e \in \mathcal{B} \cup \mathcal{D}$ and $e^{\prime} \in \mathcal{B} \cup \mathcal{D}$ such that $e \subset e^{\prime}$.

Clearly removing such $e^{\prime}$ (or moving it from the set $\mathcal{B}$ to $\mathcal{C}$ or $\mathcal{D}$ ) in case that one of the last two conditions is violated, does not affect the feasible set. If there is a $\mathcal{C}$-edge $\{u, v\}$ of size two, we can do the following: We replace the vertices $u$ and $v$ by a new vertex $w$ (i.e., we put $w$ in all the edges containing $u$ or $v$ and we remove $u$ and $v$ from the hypergraph), remove all the $\mathcal{C}$-edges containing both $u$ and $v$ from the hypergraph and change all the $\mathcal{B}$-edges containing both $u$ and $v$ to $\mathcal{D}$-edges. There is a one-to-one correspondence between proper colorings of the original and the new mixed hypergraphs ( $u$ and $v$ have to get the same color and this color can be assigned to $w$; on the other hand, the color assigned to $w$ can be assigned both to $u$ and $v$ ). Thus the feasible set of the new mixed hypergraph is the same as the feasible set of the original mixed hypergraph. Moreover, if the original mixed hypergraph is planar, then the obtained one is planar. We assume w.l.o.g. that all the mixed hypergraphs in this paper are reduced.

A weak embedding of a planar mixed hypergraph $H=(V, \mathcal{B}, \mathcal{C}, \mathcal{D})$ is an embedding of some planar multigraph with the vertex set $V$ such that each edge of $H$ corresponds to a face of the embedding (distinct edges correspond to distinct faces). Each planar mixed hypergraph has a weak embedding: Consider a planar drawing of $B(H)$. Let $e$ be an edge of $H$ and let $v_{1}, \ldots, v_{n}$ be the vertices of $e$ in the clockwise order in which they are joined to the vertex $v_{e}$ in that particular drawing of $B(H)\left(v_{e}\right.$ is the vertex of $B(H)$ corresponding to the edge $e$ ). We put new edges $v_{1} v_{2}, \ldots, v_{n-1} v_{n}, v_{n} v_{1}$ to the drawing of $B(H)$; these edges can be drawn "along" the edges $v_{i} v_{e}$ and $v_{e} v_{i+1}$. We remove all the original edges (i.e. edges of $B(H)$ ) and all the vertices corresponding to the edges of $H$ - we got a weak embedding of $H$. Note that the boundaries of all the faces in the just obtained embedding are unions of cycles.

On the other hand, the mixed hypergraph $H$ obtained from an embedding of a planar multigraph through setting some of its faces to be edges of $H$ is clearly planar: It is enough to put in each face (corresponding to an edge of $H$ ) a new vertex and join it by 
edges to the vertices of that face. After removal of all the edges of the original planar multigraph, we get a planar drawing of $B(H)$.

The planar mixed hypergraph $H$ has a strong embedding iff there exists a weak embedding such that all the faces of this embedding correspond to the edges of $H$; such embedding is called a strong embedding of $H$. A planar mixed hypergraph may not have a strong embedding.

We prove an easy lemma on the number of faces of size two in an embedding of a planar mixed hypergraph:

Lemma 1 Let $H$ be any planar mixed hypergraph different from the mixed hypergraph with two vertices forming together a $\mathcal{D}$-edge. There exists an embedding of $H$ whose number of faces of size two is the number of $\mathcal{D}$-edges of $H$ of size two and the boundaries of all its faces are unions of possibly more vertex-disjoint cycles.

Proof: Consider an embedding of $H$ obtained through the above described procedure from $B(H)$; the boundaries of its faces are unions of vertex-disjoint cycles. If there are some faces of size two not corresponding to the edges of $H$, these faces consist of two parallel edges and we can remove one of these two parallel edges from the embedding. The boundaries of all the faces remain cycles. The faces of size two can correspond only to $\mathcal{D}$-edges of $H$ after application of this procedure. This finishes the proof of the lemma.

\section{Strict Colorings of Planar Mixed Hypergraphs}

We first prove the promised generalization for planar mixed hypergraphs of the twocolorability theorem for planar hypergraphs (proved by Burshtein and Kostochka):

Theorem 1 Let $H$ be a planar mixed hypergraph satisfying the following two conditions:

- $H$ contains a $\mathcal{D}$-edge.

- $H$ contains at most two $\mathcal{D}$-edges of size two.

Then the (lower) chromatic number $\chi(H)$ of $H$ is two.

Proof: We assume w.l.o.g. that $H$ contains exactly two $\mathcal{D}$-edges of size two; we can add edges to $H$. Consider an embedding of $H$ with two faces of size two - its existence follows from Lemma 1. Triangulate all its faces of size greater than three. Let $G$ be the just obtained planar multigraph and let $H^{\prime}$ be the mixed hypergraph with $\mathcal{D}$-edges corresponding to the faces of size two of $G$ and $\mathcal{B}$-edges corresponding to the faces of size three of $G$. Any proper coloring of $H^{\prime}$ is clearly a proper coloring of $H$. We prove that $H^{\prime}$ is two-colorable. Let $c: V \rightarrow\{1,2,3,4\}$ be any four-coloring of $G$. We assume w.l.o.g. following: 
- If the two $\mathcal{D}$-edges of $H^{\prime}$ are not disjoint, i.e. they are $\{u, v\}$ and $\{u, w\}$, we assume $c(u)=1$ and $c(v), c(w) \in\{3,4\}$; otherwise we interchange the colors $c(v)$ and 3 or the colors $c(w)$ and 4 .

- If the two $\mathcal{D}$-edges of $H^{\prime}$ are disjoint, i.e. they are $\{u, v\}$ and $\{x, y\}$, we assume $c(u), c(x) \in\{1,2\}$ and $c(v), c(y) \in\{3,4\}$; otherwise, we permute the colors in order to satisfy the above conditions.

Let the coloring $\tilde{c}: V \rightarrow\{1,2\}$ of $H^{\prime}$ be defined as follows:

- $\tilde{c}(v)=1$ if $c(v)=1$ or $c(v)=2$

- $\tilde{c}(v)=2$ if $c(v)=3$ or $c(v)=4$

We claim that $\tilde{c}$ is strict proper 2-coloring of $H^{\prime}$. It is enough to prove that $\tilde{c}$ is proper; it must be strict since $H^{\prime}$ contains $\mathcal{D}$-edges. The two $\mathcal{D}$-edges of $H^{\prime}$ are colored properly due to our assumption on $c$. The remaining edges of $H^{\prime}$ are $\mathcal{B}$-edges of size three corresponding to the triangles of $G$; since its vertices are colored by $c$ with precisely three different colors, they are colored by $\tilde{c}$ with precisely two different colors - two of their vertices are colored with the same color and the last one is colored with the other color. Thus $\tilde{c}$ is a proper coloring of $H^{\prime}$.

It is not possible to allow the presence of three $\mathcal{D}$-edges of size two in the previous theorem: The triangle is a planar mixed hypergraph with three $\mathcal{D}$-edges of size two and its chromatic number is three.

We prove that planar mixed hypergraphs without edges of size two with an edge of size four have gap-free feasible sets:

Theorem 2 Let $H$ be a planar mixed hypergraph satisfying the following two conditions:

- $H$ does not contain any $\mathcal{D}$-edges of size two.

- $H$ contains at least one edge of size at least four.

Then the feasible set of $H$ is gap-free, i.e. $\mathcal{F}(H)=[\chi(H), \bar{\chi}(H)]$.

Proof: If $H$ does contain neither $\mathcal{B}$-edges nor $\mathcal{D}$-edges, then $\mathcal{F}(H)$ is clearly $[1, \bar{\chi}(H)]$. Otherwise, $\chi(H)=2$ due to Theorem 1. If we prove that $3 \in \mathcal{F}(H)$, then the feasible set of $H$ is gap-free due to the results of Kobler and Kündgen from [10] that a feasible set of a planar mixed hypergraph can contain a gap only at the number 3 (see Section 1).

We generalize ideas from $[15,21]$. Let $G$ be the embedding of $H$ from Lemma $1 ; G$ does not contain faces of size two and the boundaries of all its faces are disjoint unions of cycles. Let $G^{\prime}$ be any triangulation of $G$ and let $u v$ be one of the added edges (at least one face of $G$ has size at least four). Let $H^{\prime}$ be the (strongly-embeddable) mixed planar hypergraph with $\mathcal{B}$-edges corresponding to the faces of $G^{\prime}$. Let $G^{\star}$ be the dual of $G^{\prime}-$ $G^{\star}$ is a cubic bridgeless planar multigraph and thus it contains 2-factor due to Petersen's theorem. We distinguish two cases: 
- $G^{\star}$ contains a 2-factor which is not a Hamiltonian cycle. This 2-factor has at least three faces and its faces are 2-colorable due to trivial reasons. A 3-coloring of its faces, which clearly exists, gives 3-coloring of the vertices of $G^{\prime}$ by assigning the vertices the color of the region in which they lie. This coloring is a proper coloring of $H^{\prime}$ : Consider a triangle of $G^{\prime}$ - the two-factor splits the vertices of the triangle to two different regions; two of its vertices lie in one of these regions and the last one lies in the other region Thus each triangle of $G^{\prime}$ contains two vertices colored with the same color and one vertex colored with another color. This ensures that the coloring is a proper coloring of $H^{\prime}$. Since this coloring is a proper coloring of $H^{\prime}$, it is also a proper coloring of $H$.

- All 2-factors of $G^{\star}$ are Hamiltonian cycles. Let $C$ be any such Hamiltonian cycle; the length of $C$ is even, since $G^{\star}$ is a cubic graph. Let $e$ be the edge of $G^{\star}$ corresponding to the edge $u v$ of $G^{\prime}$. If $C$ contains the edge $e$, there is 1-factor of $G^{\star}$ containing $e$, since the length of $C$ is even. The complement of this 1-factor is a 2-factor (and thus a Hamiltonian cycle) of $G^{\star}$ omitting $e$. Thus we can further assume that $C$ omits e. Consider the subgraph $C^{\star}$ of $G^{\star}$ consisting of the Hamiltonian cycle $C$ and the edge $e$; the edges of $C^{\star}$ split the plane into three regions. Color these three regions with three different colors and color the vertices of $G^{\prime}$ (of $H^{\prime}$ ) with colors assigned to the regions in which they lie. Let $u v x$ and $u v y$ be the two faces of $G^{\prime}$ sharing the edge $u v$. All the edges of $H^{\prime}$ except for $\{u, v, x\}$ and $\{u, v, y\}$ are colored properly due to the argument used in the previous case. The edge $u v$ has been added to $G$ to get $G^{\prime}$, so the corresponding edge of $H$ contains all the four vertices $u, v, x$ and $y$. This edge clearly contains two vertices with different colors (e.g. $u$ and $v$ ). Since $C$ is Hamiltonian cycle, it splits the plane into two regions: one of them contains $u$ and $v$, the other one contains $x$ and $y$. The addition of the edge $e$ splits the region containing $u$ and $v$ into two regions putting $u$ and $v$ to its different regions. But $x$ and $y$ remain in the same region and they are colored with the same color. Thus the original edge of $H$ containing $u, v, x$ and $y$ is colored properly even in case that it is a $\mathcal{B}$-edge.

We have just proven that $H$ has a proper strict 3-coloring and thus we have finished the proof of this theorem.

The immediate corollary of the just proven theorem is following:

Corollary 1 Any strongly-embeddable planar bihypergraph has a gap-free feasible set.

Proof: If the planar bihypergraph contains an edge of size at least four, then the feasible set is gap-free due to Theorem 2. Otherwise the planar bihypergraph is three-uniform (i.e. the sizes of all its $\mathcal{B}$-edges are three) and its feasible set is gap-free due to the theorem proven in [15] (see also Section 1). 


\section{Complexity Questions}

Theorem 3 There is a polynomial-time algorithm for determining whether the lower chromatic number of a planar mixed hypergraph equals two.

Proof: Let $H$ be a given planar mixed hypergraph. If $H$ contains neither $\mathcal{B}$-edges nor $\mathcal{D}$-edges, its lower chromatic number is one. If this is not the case, we proceed as follows. We assume w.l.o.g. that $H$ does not contain any $\mathcal{C}$-edges or $\mathcal{B}$-edges (i.e. it contains only $\mathcal{D}$-edges): If $H$ contained a $\mathcal{C}$-edge of size two, we could contract it (see Section 2). If $H$ contained a $\mathcal{C}$-edge $(\mathcal{B}$-edge) of size three or more, we could remove this edge (in case of a $\mathcal{B}$-edge, we replace it by a $\mathcal{D}$-edge consisting of the same vertices) without affecting the existence of a strict 2-coloring: Any strict 2-coloring of the new mixed hypergraph is also a strict 2-coloring of the original one, since the removed (replaced) edges had size three or more and thus any 2-coloring assigns the same color to at least two of the vertices in each of the removed edges.

We construct a planar formula which is NAE-satisfiable iff $H$ has a strict 2-coloring. A formula is $N A E$-satisfiable iff there is a variable assignment which satisfies the formula and each its clause contains both a true and a false literal (i.e. not all the literals of the clause are true). A formula $\Phi$ is planar if its bipartite incidence graph $B(\Phi)$ is planar: The vertices of $B(\Phi)$ are the variables and the clauses of $\Phi$ and a variable is joined by an edge to a clause iff the variable is contained in that clause. Determining whether a planar formula is NAE-satisfiable is solvable in polynomial time: there is a polynomial reduction of NAE-satisfiability of planar formulae, as stated in [14], to another polynomial-time solvable problem of determining maximum edge-cut for planar graphs (see [8]).

We introduce for each vertex $v$ of $H$ a variable $x_{v}$. We form clauses corresponding to the edges of $H$ : We form the clause $\left(\bigvee_{v \in e} x_{v}\right)$ for each $(\mathcal{D}-$ )edge $e$ of $H$. The resulting formula $\Phi$ is clearly planar (since $B(\Phi)=B(H)$ ) and it is NAE-satisfiable iff $H$ has a strict 2-coloring: Coloring the vertices of $H$ with two colors can be translated to the truth assignment to the variables of $\Phi$ by assigning true to the vertices colored with one of the two colors and false to the vertices colored with the other color. On the other hand, each variable assignment of $\Phi$ can be translated to a 2-coloring of $H$ similarly. The variable assignment of $\Phi$ is clearly NAE-satisfying iff the corresponding coloring of $H$ is proper. This finishes the proof of the theorem.

We summarize the results about the complexity of determining the lower chromatic number in the following corollary (note that the lower chromatic number of a planar mixed hypergraph cannot exceed 4):

Corollary 2 The following holds for the problem of determining whether the lower chromatic number of a given planar mixed hypergraph equals a fixed number $k$ :

- If $k=1$, the problem is solvable in polynomial time.

- If $k=2$, the problem is solvable in polynomial time. 
- If $k=3$, the problem is NP-complete.

- If $k=4$, the problem is coNP-hard.

Proof: Since the lower chromatic number of a mixed hypergraph equals one iff it contains only $\mathcal{C}$-edges, the first statement is trivial. The second statement is due to Theorem 3 and the third one and the fourth one are due to the well-known result that even the decision problem whether a planar graph can be colored by at most three colors is NP-complete (see [6]).

Lemma 2 It is NP-complete to decide whether a planar bridgeless multigraph contains a 2 -factor with at least $k$ components where $k$ is part of input.

Proof: We present a reduction from a planar satisfiability problem which is known to be NP-complete due to [17]. See the proof of Theorem 3 for the definition of a planar formula. We may assume w.l.o.g. (see [5]) that the input formula $\Phi$ satisfies the following:

- The planar graph $B(\Phi)$ is connected.

- Each variable occurs in exactly three clauses, twice as a positive literal (i.e. $x$ ) and once as a negative literal (i.e. $\bar{x}$ ).

- Each clause has size either two or three.

- No clause contains two occurrences of the same variable.

We replace each variable with the gadget from Figure 1, each clause of size two with the gadget from Figure 2 and each clause of size three with the gadget from Figure 3 . We identify the corresponding red vertices of the clause gadgets and of the variable gadgets (i.e. the vertex of the clause corresponding to the positive/negative occurrence of the variable). We claim that the resulting planar bridgeless graph has a 2-factor with at least $c_{2}+3 c_{3}+2 v$ components where $c_{2}$ is the number of clauses of size two, $c_{3}$ is the number of clauses of size three and $v$ is the number of variables of $\Phi$, iff the formula $\Phi$ is satisfiable.

We first prove that if $\Phi$ is satisfiable, then the planar multigraph has a 2-factor with $c_{2}+3 c_{3}+2 v$ components. We choose for each clause one of possibly more literals which make that clause satisfied; we call this occurrence of the variable important. Note that for each variable $x$ precisely one of the following statements holds:

- The variable $x$ has no important occurrences.

- The variable $x$ has exactly one positive important occurrence.

- The variable $x$ has exactly two positive important occurrences.

- The variable $x$ has exactly one negative important occurrence. 

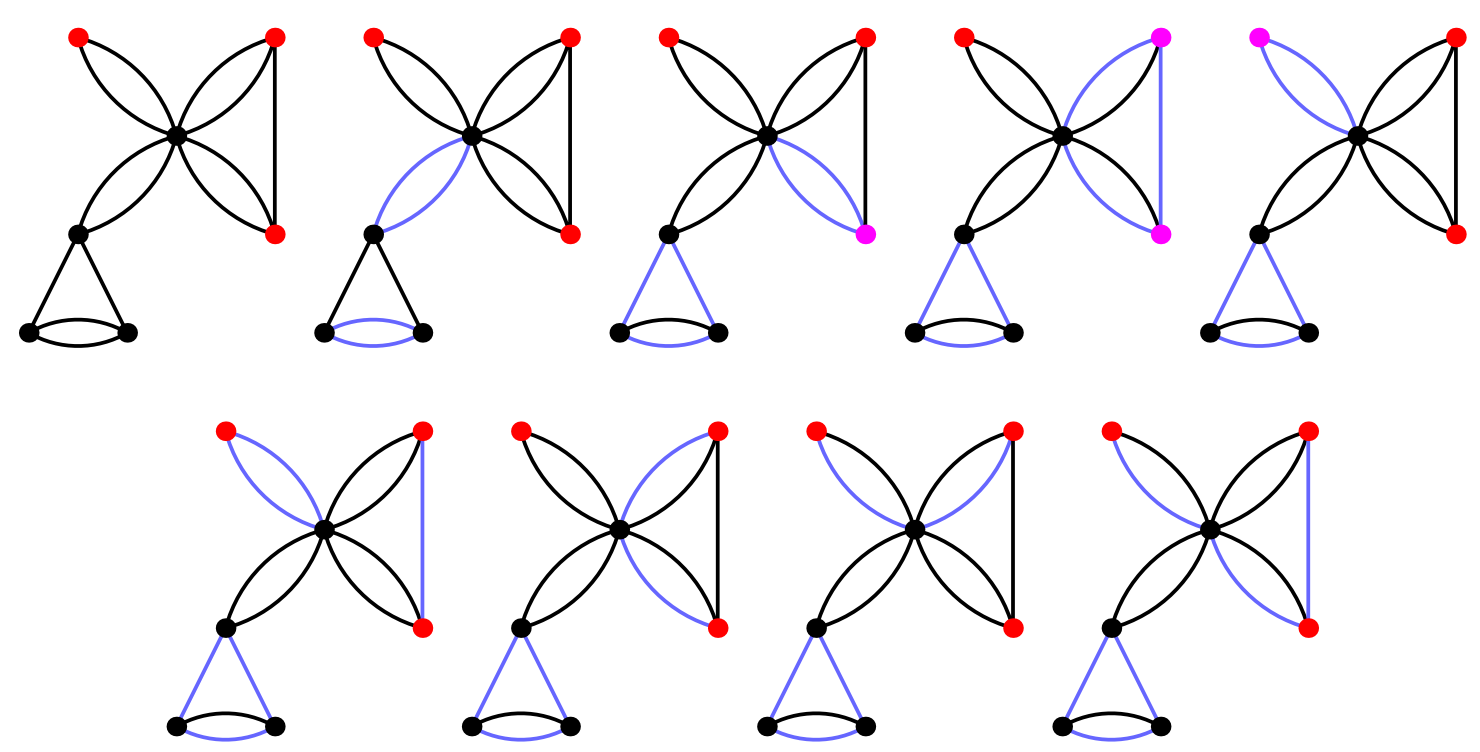

Figure 1: The variable gadget for the variable $x$ and possibilities of containing edges in a 2 -factor. The right contact vertices correspond to the positive occurrences of the variable $(x)$ and the left one corresponds to the negative occurrence of the variable $(\bar{x})$. Several possibilities of containing edges in a 2-factor which are actually the same as one of those in the figure are omitted. The contact vertices are marked by red, the important occurrences by magenta and the edges of a 2 -factor by blue.
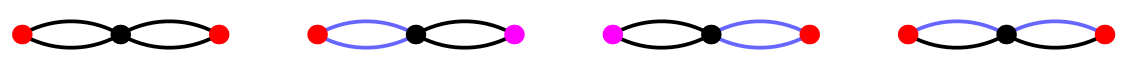

Figure 2: The clause gadget for a clause of size two and possibilities of containing edges in a 2-factor. Three possibilities which are actually the same as the right one are omitted. The contact vertices are marked by red, the important occurrences by magenta and the edges of a 2-factor by blue. 

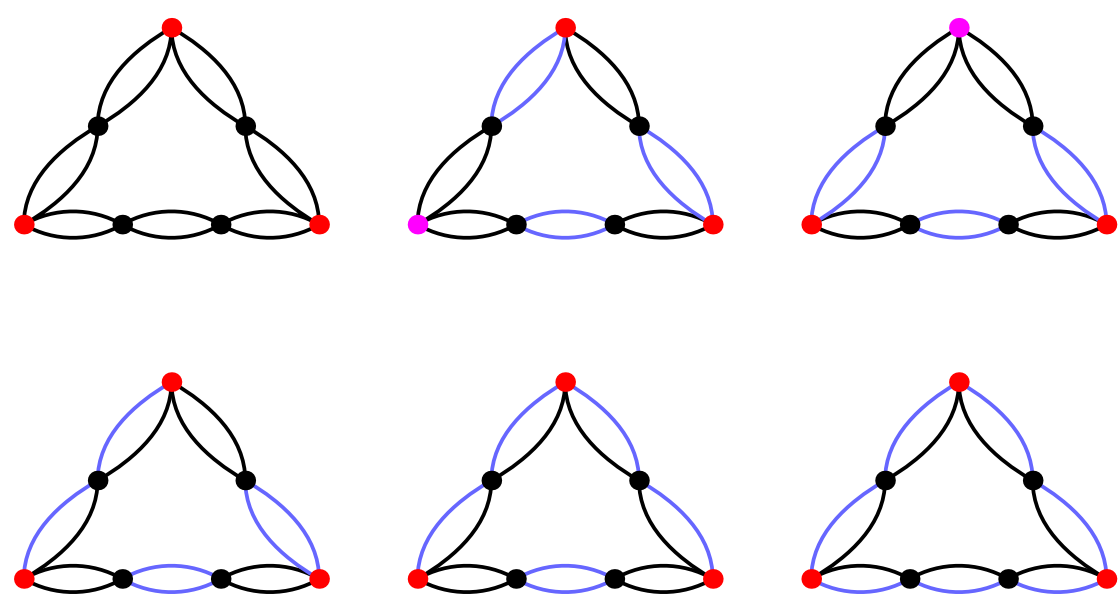

Figure 3: The clause gadget for a clause of size three and possibilities of containing edges in a 2-factor. One possibility which is actually the same as the middle top one and several possibilities which are actually the same as one of the bottom ones are omitted. The contact vertices are marked by red, the important occurrences by magenta and the edges of a 2 -factor by blue.

We construct the desired 2-factor as follows: The gadget of a clause of size two (three) contains the whole one (three) components of the 2-factor in such way that the components omit the important occurrence of the variable, i.e. the 2-factor restricted to that gadget is one of the two middle ones in Figure 2 or one of the two right top ones in Figure 3. The 2-factor restricted to the variable gadget is one of the four right top ones in Figure 1; the components which are whole in the variable gadgets contain all the vertices corresponding to the important occurrences (magenta ones in Figure 1). The order of the possible restriction of the 2-factor in Figure 1 is the same as the order in which the possibilities of number and type of important occurrences of the variable are listed above. The just obtained 2-factor consist of $c_{2}+3 c_{2}+2 v$ components.

We prove the opposite implication in the next two paragraphs. Suppose that the graph contains a 2 -factor with at least $c_{2}+3 c_{3}+2 v$ components. In the next paragraph, we prove that then the 2-factor restricted to the gadgets is one of the top ones in Figure 1 and Figure 3 or one of two middle ones in Figure 2. This implies that it consists of exactly $c_{2}+3 c_{3}+2 v$ components and it establishes the correspondence between any 2-factor with $c_{2}+3 c_{3}+2 v$ components and the satisfying variable assignments actually in the same way as described for the opposite direction in the previous paragraph.

Let $F$ be a 2 -factor consisting of at least $c_{2}+3 c_{3}+2 v$ components. We count how many components of $F$ each of the gadgets contains fully and how many partially. The variable gadget (consult Figure 1) can contain:

- two of the components fully and none of them partially.

- two of the components fully and one of them partially.

- one of the components fully and one of them partially. 
The clause gadget of a clause of size two (consult Figure 2) can contain:

- fully one of the components and none of them partially

- none of the components fully and one of them partially.

The clause gadget of a clause of size three (consult Figure 3) can contain:

- three of the components fully and none of them partially.

- two of the components fully and one of them partially.

- one of the components fully and one of them partially.

- one of the components fully and none of them partially.

Each component of $F$ contained in more gadgets is contained in at least two clause gadgets, since otherwise one of the clauses of the original formula would contain two occurrences of the same variable. We use charging method to count the number of the components of $F$. Each component gives at least one unit together to all the gadgets which it is contained in; thus the sum after charging is at least the number of the components of $F$. Each component fully contained in one of the gadgets gives one unit to that gadget; each other component gives one half of the unit to each of the clause gadgets which it is partially contained in. No variable gadget gets more than two units, no clause gadget of a clause of size two gets more than one unit and no clause gadget of a clause of size three gets more than three units. If $F$ consists of $c_{2}+3 c_{3}+2 v$ components, then each of the gadgets gets the maximum possible number of units (they have to get altogether at least $c_{2}+3 c_{3}+2 v$ units). Because of this no clause gadget can contain any of the components of $F$ partially and thus also no variable gadget can. This implies that each of the components of $F$ is fully contained in one of the gadgets and thus $F$ restricted to the gadgets looks like claimed in the previous paragraph.

Lemma 3 It is NP-complete to decide whether a 3-regular planar bridgeless graph contains a 2-factor with at least $k$ components where $k$ is part of input.

Proof: We continue the reduction presented in the proof of Lemma 2. We use instead of the variable gadget from Figure 1, the gadget from Figure 4. Since we only replaced two parallel edges by three ones, the structure of 2-factors is not affected and the reduction proceeds as described in the proof of Lemma 2. This small change allows to pair the edges incident to the vertices as marked by different colors in Figure 5 and it allows us to assume w.l.o.g. that a 2 -factor with $c_{2}+3 c_{3}+2 v$ components (if it exists) contains at each vertex only the edges incident to it with the same color. Note that the edges are paired in such way that paired edges are always the neighboring (in clockwise order) edges incident to the vertex. 


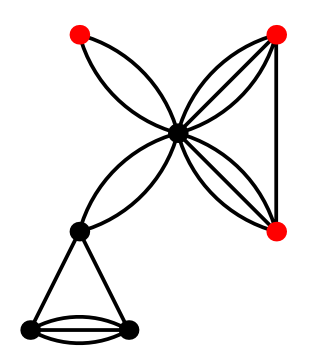

Figure 4: The variable gadget for the reduction of the proof of Lemma 3.
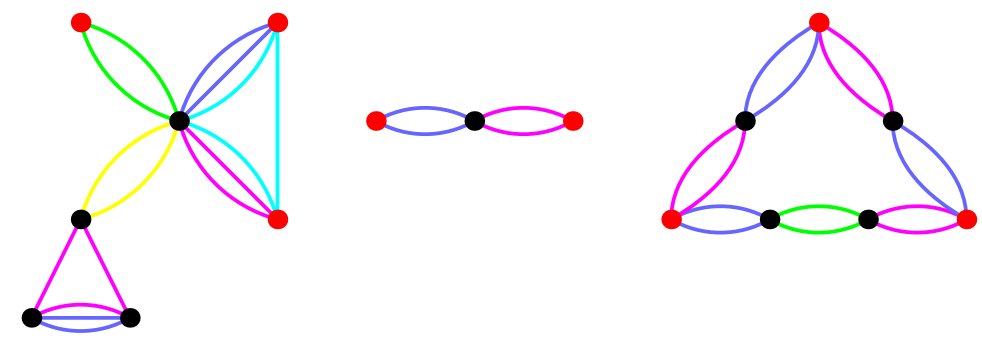

Figure 5: Pairing the edges incident to the vertices in the gadgets. The pairs of the edges incident to the vertices are marked by different colors. We may assume w.l.o.g., as explained in the proof of Lemma 3, that each 2-factor with maximum possible number of components contains at each vertex only the edges with the same color.

The planar bridgeless graph from the reduction contains vertices of degree four, six, eight and ten - we replace each of the vertices by a gadget consisting of vertices of degree 3 with the following property: Any 2-factor of the new graph after contraction of the gadgets to the vertices is a 2-factor of the original graph; on the other hand, any 2 -factor of the original graph which uses at each vertex the two edges of the same color corresponds to a 2-factor of the new graph. We first allow a presence of special edges (the edges are bold red ones in the figures) which we demand to be a part of any 2factor; we later replace these special edges by suitable gadgets. The vertices of degree four are replaced by the gadget from Figure 6 . It is clear that this gadget has the claimed properties. The vertices of degree six are replaced by the gadget from Figure 7 . In a 2 -factor, the central vertex is adjacent to two of its edges and it omits one of them; suppose that it omits the top edge (as drawn in Figure 7), then the vertex immediately above it has to form a triangle (the very top edge is a special one). Each of the edge

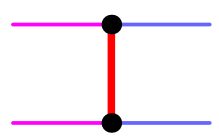

Figure 6: The gadget replacing a vertex of degree four. The paired edges are marked by blue and by magenta color; the special edge which has to be used in a 2-factor is marked by red color. 

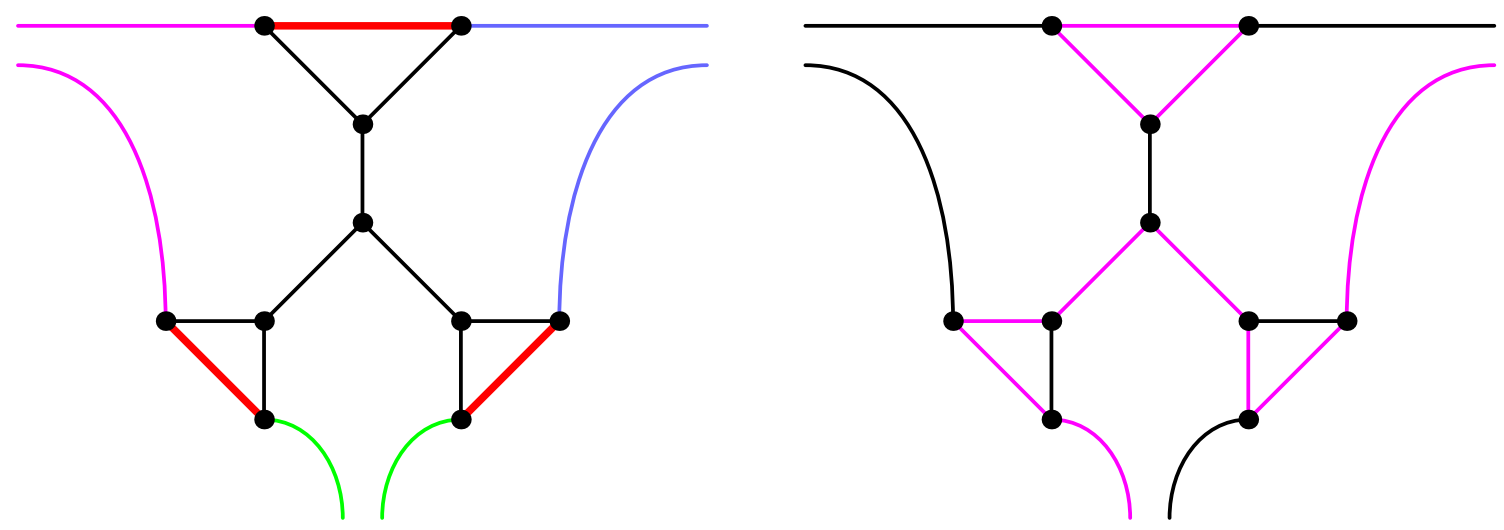

Figure 7: The gadget replacing a vertex of degree six. The paired edges are marked by blue, magenta and green color; the special edge which has to be used in a 2-factor is marked by red color. How the restriction of a 2-factor to the gadget could be like is marked by magenta color in the right.

of the 2-factor incident to the central vertex is connected out of the gadget by a path consisting of a 2-factor edges (there are four possibilities of this if the top edge incident to the central vertex is omitted; one of these possibilities is in Figure 7). Thus the gadget has the claimed properties and it increases the number of components of a 2-factor by exactly one. The vertices of degree 8 and 10 of the original graph are first replaced by vertices of degree six (see Figure 8) and then the new vertices of degree six are replaced by the gadgets from Figure 7. It is straightforward work to check that all the gadgets have the claimed properties and it is left to the reader. If the maximum possible number of components of a 2 -factor of the original graph was $k$, then the maximum possible number of components of the obtained graph is $k+d_{6}+3 d_{8}+5 d_{10}$ where $d_{6}$ (respectively $d_{8}, d_{10}$ ) is the number of vertices of degree 6 (respectively 8,10 ) of the original graph. The obtained graph is planar, 3-regular and bridgeless. Unfortunately, it contains special edges.

We have to get rid of the special edges. Let $G$ be the obtained (planar 3-regular bridgeless) graph with special edges, let $n$ be the number of its vertices, let $m$ be the number of its special edges and let $k$ be the maximum possible number of components of a 2-factor of $G$ which contains all the special red edges. We replace each red edge with the gadget from Figure 9; the gadget is inserted into the edge, i.e. the original ends of the edge are marked in Figure 9 by red color. The number of diamonds in the top part of the gadget equals $n$ (the number of vertices of $G$ ). Let $G^{\prime}$ be the resulting graph. Note that $G^{\prime}$ is 3-regular bridgeless planar graph. The number of vertices of $G^{\prime}$ is $n+m(4 n+2)$ and we claim that the maximum possible number of components of a 2 -factor of $G^{\prime}$ is $k+m n$. The claim implies that the original problem whether $G$ contains a 2 -factor containing all the special edges consisting of at least $k$ components is equivalent to the problem whether $G^{\prime}$ contains a 2 -factor consisting of at least $k+m n$ components, i.e. the maximum possible number of components of a 2-factor of $G$ containing all the special edges is $k$ iff the maximum possible number of components of a 2 -factor of $G^{\prime}$ is $k+m n$. If $G$ does not contain any 2 -factor containing all the special edges, then the maximum 

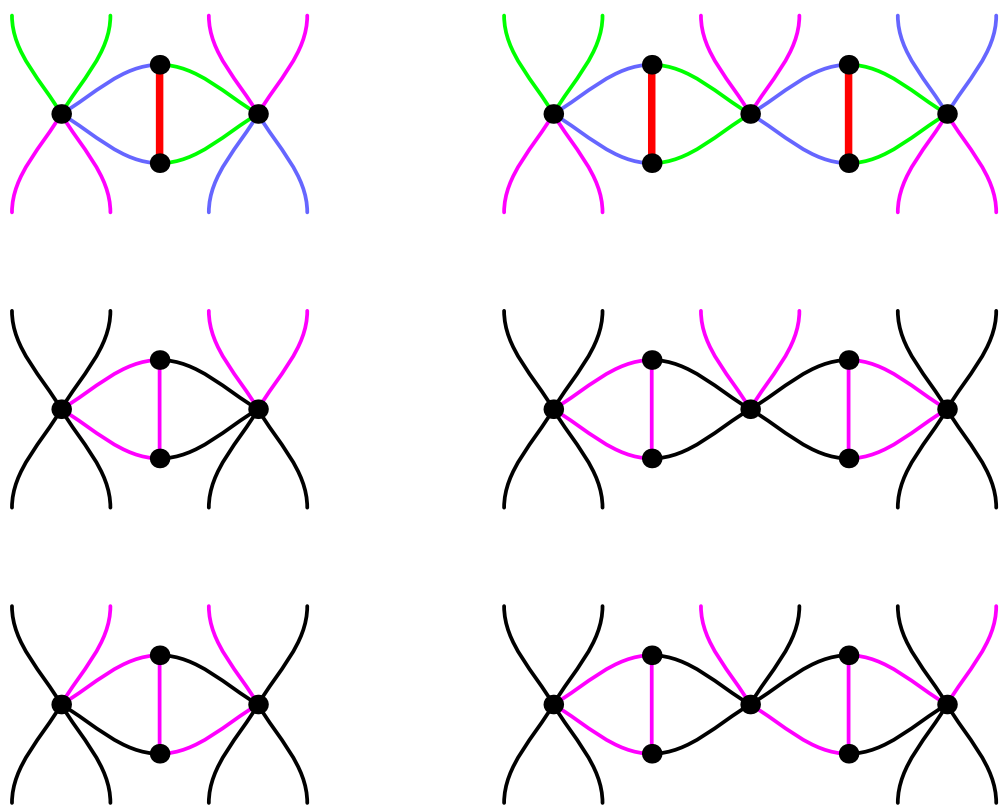

Figure 8: Replacements of vertices of degree 8 and 10 are shown in the top line; the special edges which have to be part of each 2 -factor are marked by red color. The replacements are inserted in the graph in such way that the edges incident to the vertex are replaced by the unterminated edges in the figures and the paired incident edges are preserved. The paired edges are marked by blue, magenta and green color. Some of possible restrictions of a 2 -factor are in the middle and bottom line marked by magenta color. 

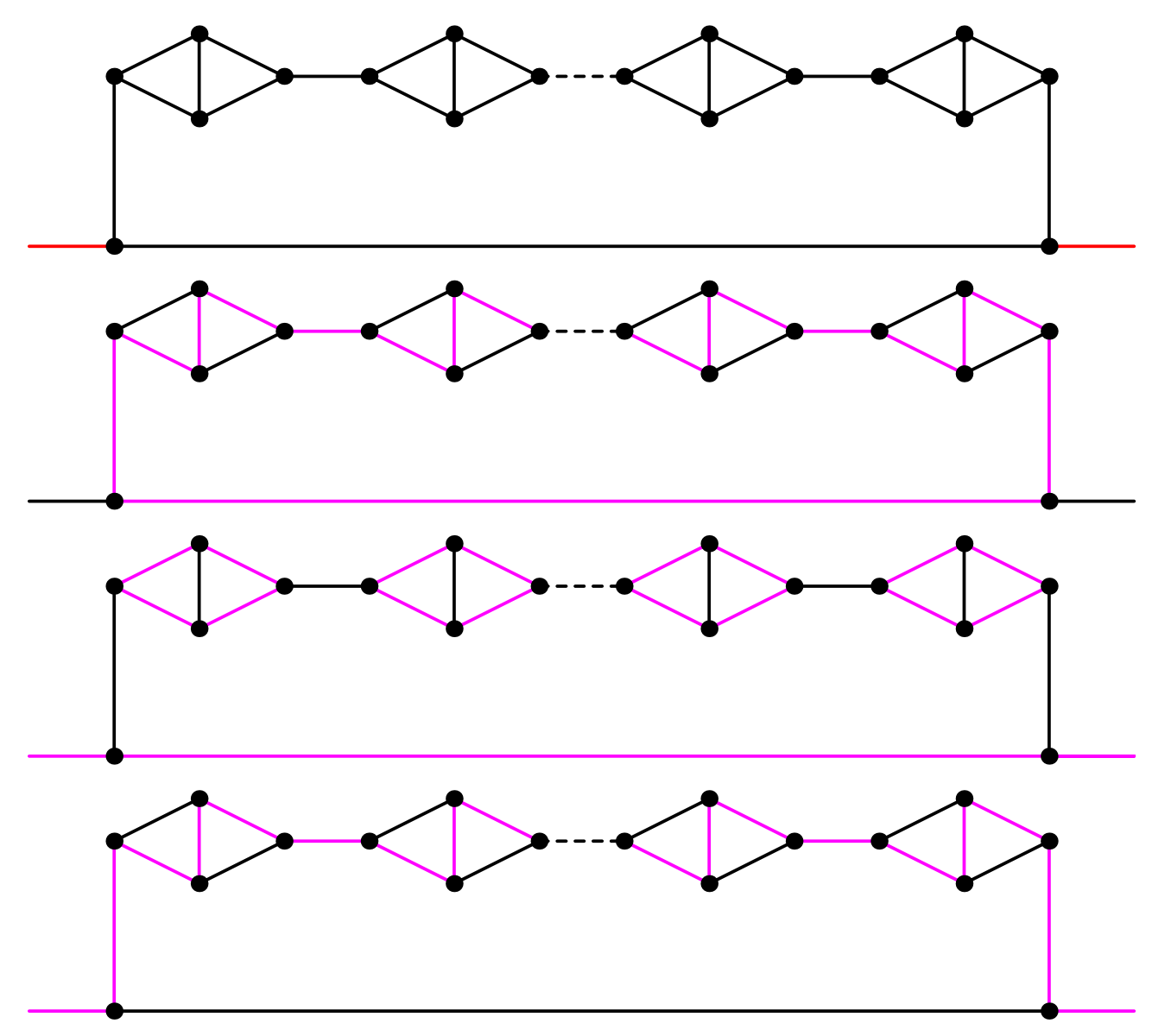

Figure 9: The gadget replacing a special red edge and the three essential possibilities of restriction of a 2-factor to the gadget (the edges of a 2-factor are marked by magenta color); note that the last one cannot be a part of a 2-factor with maximum possible number of components. The gadget is pasted into the edge (the original ends of the edge are marked by red color). The number of diamonds equals the number of vertices of the original graph.

possible number of components of a 2 -factor of $G^{\prime}$ is at most $m n$. Proving this claim finishes the proof of the lemma. Note that all the transformations described during the proof are polynomial-time.

Let $F^{\prime}$ be a 2 -factor of $G^{\prime}$ with maximum possible number of components. We remove all the gadgets corresponding to the red edges of $G$, we replace them back by edges. Since either both the connecting edges of the gadget are contained in $F^{\prime}$ or both are not contained in $F^{\prime}, F^{\prime}$ arises a 2-factor $F$ of $G$ (consult Figure 9 ). Let $m^{\prime}$ be the number of special edges contained in $F$; if the number of components of $F$ is $k$, then the number of components of $F^{\prime}$ is $k+m^{\prime} n$. Since $k<n$ (the number of components of a 2-factor of $G$ cannot exceed its number of vertices), if $k+m^{\prime} n$ is greater than $m n$ than $m^{\prime}$ has to be $m$ and $F$ cannot omit any special edge. Thus if $G^{\prime}$ contains a 2-factor with $k+m n$ components, then $G$ contains a 2 -factor with $k$ components which contains all the special 
edges. On the other hand, a 2-factor $F$ of $G$ containing all the special edges which consists of $k$ components can be extended to a 2-factor $F^{\prime}$ of $G^{\prime}$ consisting of $k+m n$ components by including the $n$ diamonds of each of the gadgets (as drawn in the middle in Figure 9). This finishes the proof of the claim and thus also the proof of the whole lemma.

The following lemma which was proved in [15] allows us to extend the reduction of Lemma 3 to determining the upper chromatic number of a strongly-embeddable 3-regular planar bihypergraph:

Lemma 4 Let $H$ be a strongly-embeddable 3-regular planar bihypergraph and let $G$ be any of its strong embedding in the plane. Let $G^{\star}$ be the dual graph of $G$. Then the maximum possible number of components of a 2 -factor of $G^{\star}$ equals $\bar{\chi}(H)-1$.

We prove the main theorem using Lemma 3 and Lemma 4:

Theorem 4 It is NP-complete to decide whether the upper chromatic number of a planar mixed hypergraph is at least given $k$ even for strongly-embeddable 3-uniform planar bihypergraphs such that their strong embeddings do not contain parallel edges.

Proof: Let $G$ be any instance of the problem of determining whether a 3-regular bridgeless planar graph contains a 2 -factor with at least $k$ components and let us suppose that $G$ is embedded to the plane. Unfortunately the dual graph of $G$ can contain parallel edges. We get rid of the parallel edges of its dual graph.

Let $n$ be the number of vertices of $G$ and let $m=3 n / 2$ be the number of its edges. We replace each edge with the gadget from Figure 10 (the ends of the original edge are marked by red color); each "ladder" consists of $2 n$ blue edges. The "ladders" of all the edges incident to the same face of $G$ are terminated on a cycle in the middle of each face as shown in Figure 11. Let $G^{\prime}$ be the obtained graph. $G^{\prime}$ is clearly 3-regular, planar and bridgeless. Moreover, $G^{\prime}$ does not contain two faces sharing two (or more) edges and thus its dual graph does not contain parallel edges. We claim that the maximum possible number of components of a 2-factor of $G^{\prime}$ is $k+2 m(n+2)=k+3 n^{2}+6 n$ where $k$ is the maximum possible number of components of a 2 -factor of $G$. The prove of this claim will finish the proof of the theorem: We consider the dual graph of $G^{\prime}$ and we set all its faces (they are triangles, since $G^{\prime}$ is 3 -regular) to be $\mathcal{B}$-edges. Since $G^{\prime}$ is bridgeless and no two of its faces share two (or more) edges, its dual contains neither loops nor parallel edges. The upper chromatic number of the obtained strongly-embeddable 3uniform planar bihypergraph is equal to the maximum possible number of components of a 2 -factor of $G^{\prime}$ increased by one due to Lemma 4 . This proves that the decision problem whether the upper chromatic number of a given 3-uniform strongly-embeddable planar bihypergraph is at least a given number is NP-complete.

We prove the above claim in this paragraph. The maximum possible number of components of a 2 -factor of $G^{\prime}$ is at least $k+3 n^{2}+6 n$, since if we consider any 2-factor of $G$ we can extend it by adding exactly 2 components in the gadget and $n$ components in 


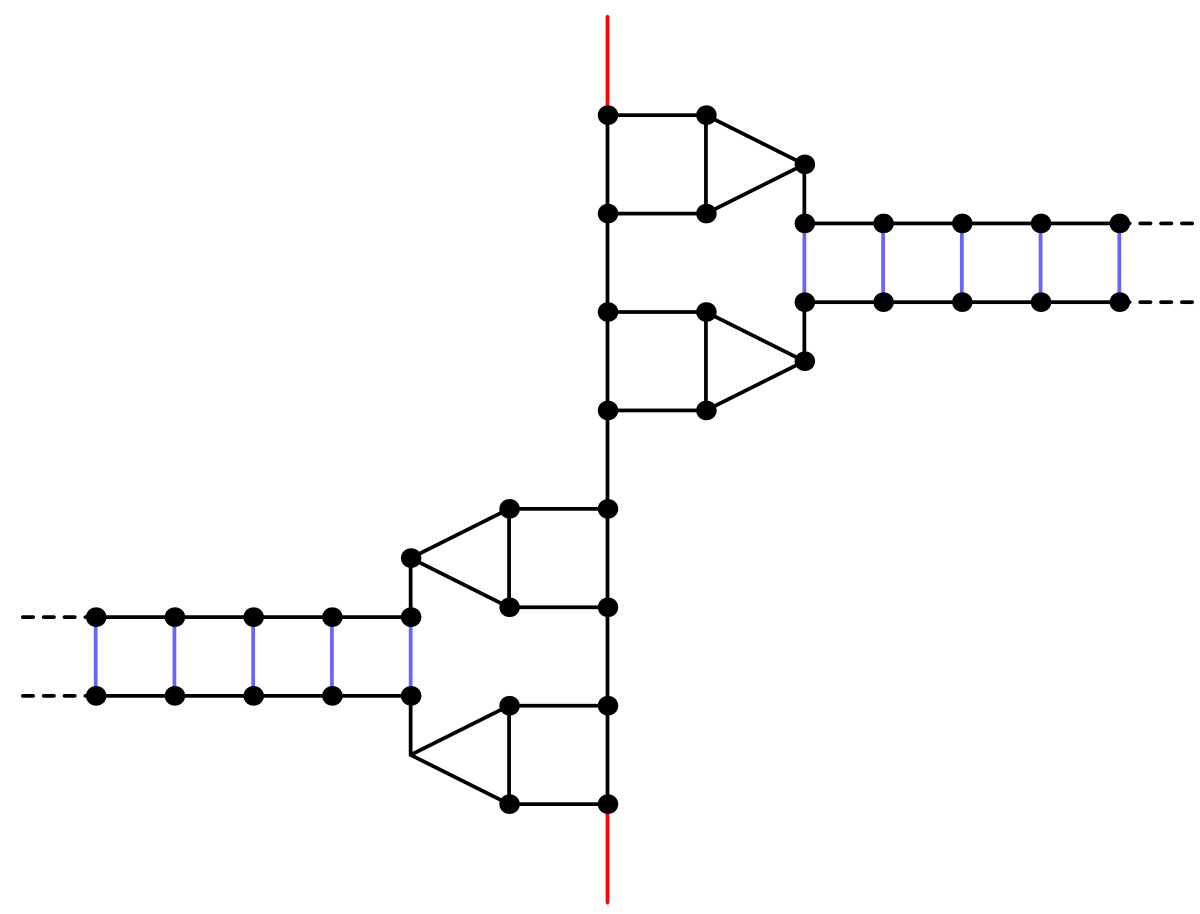

Figure 10: The gadget replacing each edge of the graph in the construction in the proof of Theorem 4. The ends of the original edge are marked by red color. The number of blue edges in each "ladder" is twice the number of vertices of the input graph.
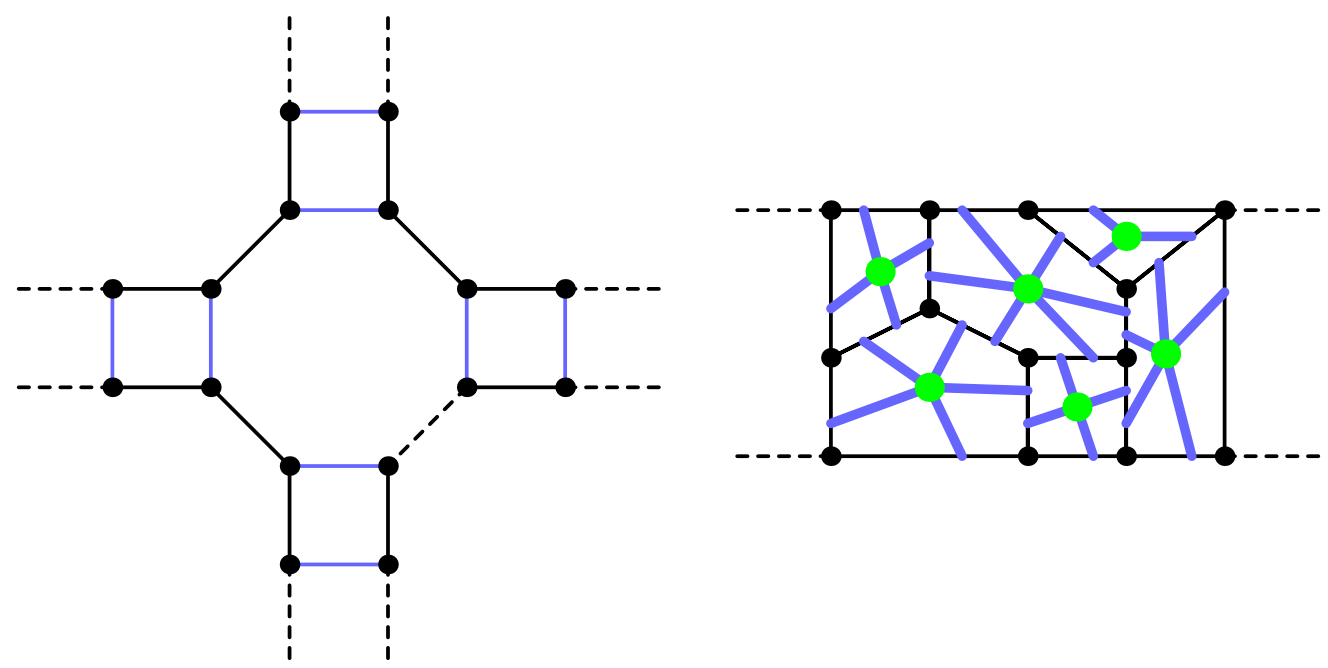

Figure 11: Termination of the "ladders" (consisting of blue edges) in the middle of the faces. Part of the input graph after inserting the gadgets is sketched in the right. The input graph is drawn by black color, the "ladders" by blue and the central cycles are marked by green circles. 

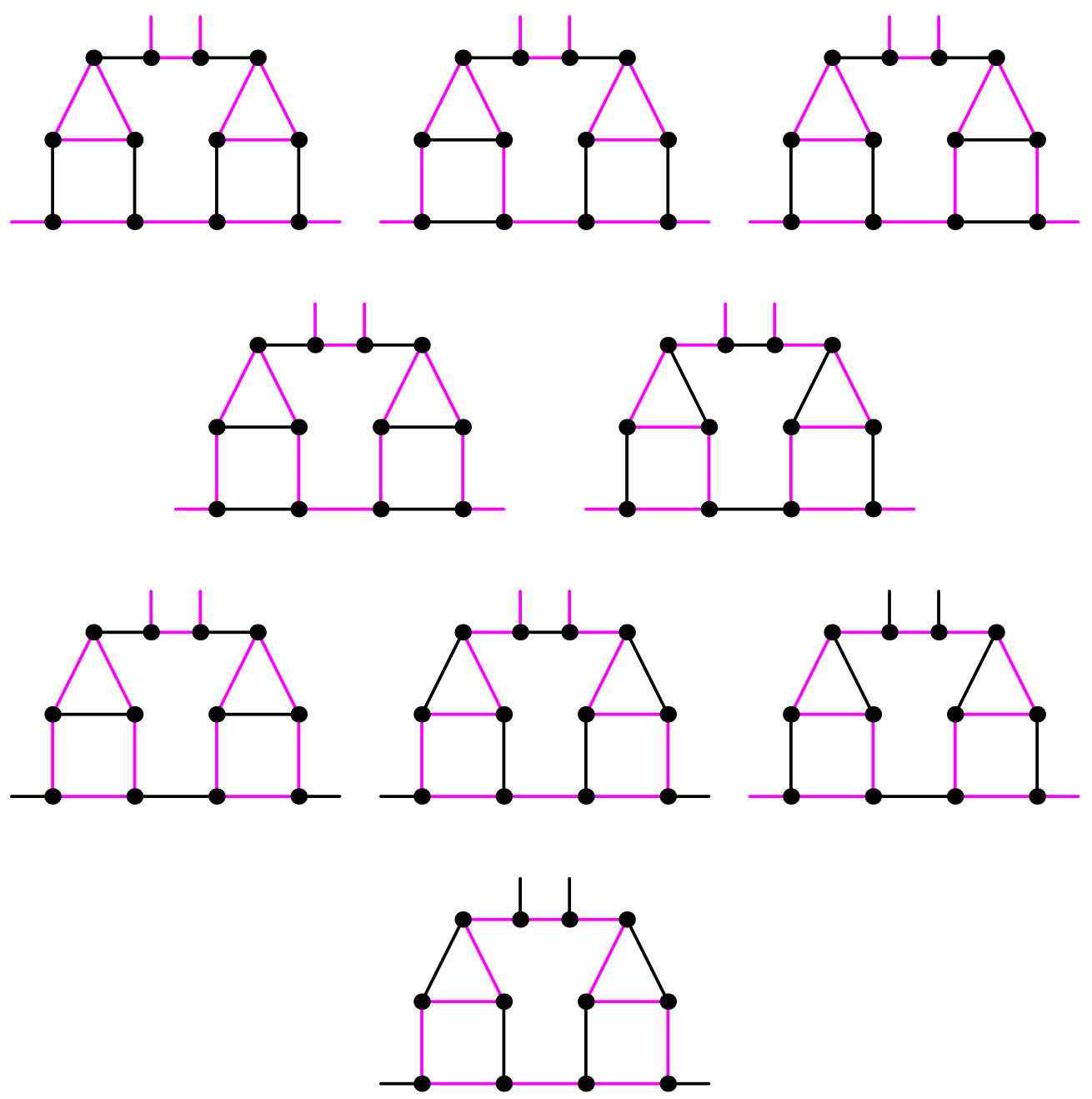

Figure 12: Possible restrictions of a 2-factor to the gadget introduced in Figure 10. The edges of a 2-factor are marked by magenta color. The first two lines contain such restrictions that the gadget contains two components of the 2-factor partially, the third line those ones that the gadget contains one component partially and the last fourth line that one that the gadget contains none of the components partially. Those restrictions in which a component of the 2-factor has to "go" through the ladder are omitted (consult the proof of Theorem 4). 

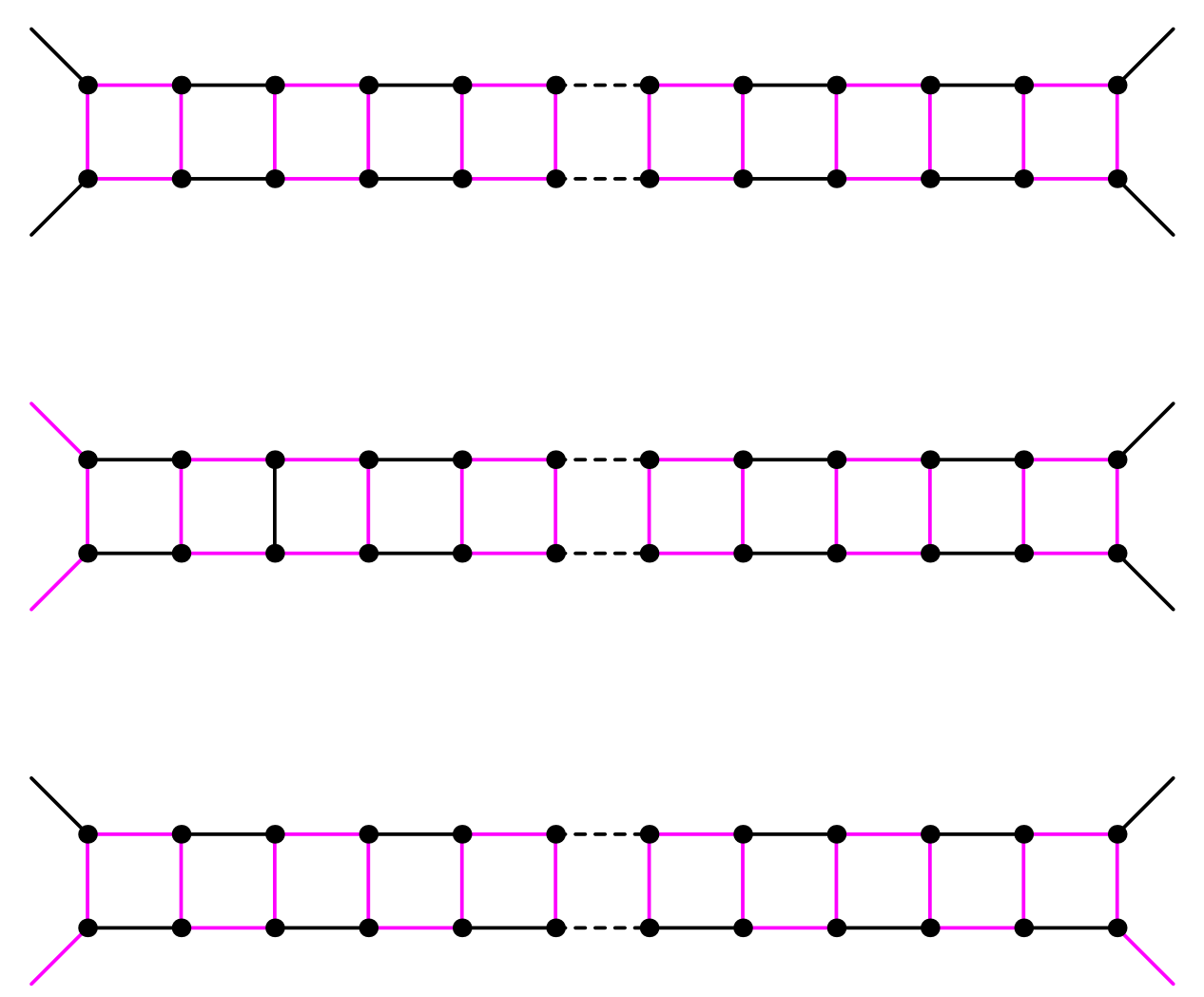

Figure 13: Some of possible restrictions of a 2-factor to a "ladder". The edges of a 2-factor are marked by magenta color.

each ladder - the restriction to the gadgets of the extended 2-factor is like the first one on the first line or the first one on the third line in Figure 12 and its restriction to each of the ladders consists of $n$ 4-cycles as shown in the top in Figure 13. Thus this extension increases the number of the components of the 2 -factor by $2 m(n+2)=3 n^{2}+6 n$. Let $F$ be further a 2 -factor of $G^{\prime}$ with the maximum possible number of components and let the number of its components be $k^{\prime}+3 n^{2}+6 n$. Since $F$ have more than $2 m(n+2)$ components and it is a 2 -factor with maximum number possible number of components, the restriction of $F$ to each of the "ladders" consists of 4-cycles and at most one 6-cycle, in particular no component of $F$ "goes through" any "ladder" as shown in the bottom of Figure 13. Thus the restriction of $F$ to any of the gadgets looks like one of those in Figure 12. Since $F$ has maximum number of components, these restrictions have to be either the first one on the first line or the first one on the third line (otherwise we could increase the number of components of $F$ by modification of its structure in the gadgets and the "ladders" of one face). But then, if we remove the "ladders" and replace the gadgets with the original edges, we get a 2 -factor of the original graph $G$ with $k^{\prime}$ components and thus $k^{\prime} \leq k$, i.e. the number of components of $F$ cannot exceed $k+3 n^{2}+6 n$. This finishes the proof of the claim. 


\section{Conclusion}

We have investigated some properties of the planar mixed hypergraphs, but some problems related to them still remain open.

We have extended the result from [15] that the feasible set of any strongly-embeddable 3-uniform planar bihypergraph is gap-free to all strongly-embeddable planar bihypergraphs. The next possible extension could be to all planar bihypergraphs:

- Is it true that the feasible set of any planar bihypergraph is gap-free?

We proved that the feasible set of any planar mixed hypergraph without edges of size two with an edge of size at least four is gap-free. It remains unclear whether the presence of edges of size two is essential for the feasible set to be broken:

- Is it true that the feasible set of any planar mixed hypergraph without edges of size two is gap-free?

Note that the gap can be only at the number 3 due to the results of [10]. There is a conjecture that $K_{4}$ is the only planar cubic graph whose all 2-factors are Hamiltonian cycles (see [15]). The proof of this conjecture would give the proof of strict 3-colorability for almost all the planar mixed hypergraphs without edges of size two (see the proof of Theorem 2 and consult $[10,15])$ and thus it would prove that the feasible sets of all such planar mixed hypergraphs are gap-free.

We proved that it is NP-complete to determine whether the upper chromatic number of a planar mixed hypergraph is at least a given number even for strongly-embeddable 3-uniform planar bihypergraphs such that their embeddings do not contain parallel edges. It remains open how difficult this problem is when the number of colors is fixed:

- What is the complexity of the problem to determine whether the upper chromatic number of a given planar (bi)hypergraph is at least a fixed number?

\section{Acknowledgement}

The authors are grateful to Jan Kratochvíl for attracting their attention to mixed hypergraphs and discussions on the topic.

\section{References}

[1] C. Berge: Graphs and Hypergraphs, North Holland, 1973.

[2] C. Colbourn, J. Dinitz, A. Rosa: Bicoloring Triple Systems, Electronic J. Combin. 6 (1), 1999, R25, 16 pages.

[3] C. J. Colbourn, A. Rosa: Triple Systems, Clarendon Press, Oxford, 1999, sect. 18.6. Strict colouring and the upper chromatic number, 340-341. 
[4] T. Etzion and A. Hartman: Towards a large set of Steiner quadruple systems, SIAM J. Discrete Math. 4 (1991), 182-195.

[5] M. R. Fellows, J. Kratochvíl, M. Middendorf, F. Pfeiffer: The complexity of induced minors and related problems, Algorithmica 13, 1995, 266-282.

[6] M. R. Garey, D. S. Johnson: Computers and Intractability, A Guide to the Theory of NP-completeness, Freeman, San Franscisco, Cal., 1979.

[7] M. R. Garey, D. S. Johnson, R. E. Tarjan: The Planar Hamiltonian Circuit Problem is NP-Complete, SIAM J. Comput., 5(4), 1976, 704-714.

[8] F. Hadlock: Finding a Maximum Cut of a Planar Graph in Polynomial Time, SIAM J. Comput. 4(3), 1975, 221-225.

[9] T. Jiang, D. Mubayi, Zs. Tuza, V. Voloshin and D. B. West: Chromatic spectrum is broken, 6th Twente Workshop on Graphs and Combinatorial Optimization, 26-28, May, 1999, H. J. Broersma, U. Faigle and J. L. Hurink (eds.), University of Twente, May, 1999, 231-234.

[10] D. Kobler, A. Kündgen: Gaps in the chromatic spectrum of face-constrained plane graphs, Electronic J. Combin. 3, 2001, \#N3.

[11] D. Král': On Complexity of Colouring Mixed Hypertrees, Proceedings 13th International Symposium on Fundamentals of Computing Theory, 1st International Workshop on Efficient Algorithms, LNCS vol. 2138, 2001, 516-524.

[12] D. Král', J. Kratochvíl, A. Proskurowski, H.-J. Voss: Coloring mixed hypertrees, Proceedings 26th Workshop on Graph-Theoretic Concepts in Computer Science, LNCS vol. 1928, 2000, 279-289.

[13] D. Král', J. Kratochvíl, H.-J. Voss: Complexity Note on Mixed Hypergraphs, Proceedings 26th International Symposium on Mathematical Foundations of Computer Science, LNCS vol. 2136, 2001, 474-486.

[14] J. Kratochvíl, Zs. Tuza: On the complexity of bicoloring clique hypergraphs of graphs, extended abstract, 11th Annual ACM-SIAM Conference on Discrete Algorithms, SODA 2000, 40-41.

[15] A. Kündgen, E. Mendelsohn, V. Voloshin: Colouring planar mixed hypergraphs, Electronic J. Combin. 7, 2000, \#R60.

[16] H. Lefmann, V. Rödl, and R. Thomas: Monochromatic vs. multicolored paths, Graphs Combin. 8 (1992), 323-332.

[17] D. Lichtestein: Planar formulae and their uses, SIAM J. Comput. 11, 1982, 329-343. 
[18] L. Milazzo: On upper chromatic number for SQS(10) and SQS(16), Le Matematiche L (Catania, 1995), 179-193.

[19] L. Milazzo and Zs. Tuza: Upper chromatic number of Steiner triple and quadruple systems, Discrete Math. 174 (1997), 247-259.

[20] L. Milazzo and Zs. Tuza: Strict colorings for classes of Steiner triple systems, Discrete Math. 182 (1998), 233-243.

[21] J. G. Penaud: Une propriété de bicoloration des hypergraphes planaires (in French), Colloque sur la Théorie des Graphes, Paris, 1974, Cahiers Centre Études Recherche Opér. 17, 1975, 345-349.

[22] V. Voloshin: The mixed hypergraphs, Computer Science Journal of Moldova 1, 1993, $45-52$.

[23] V. Voloshin: On the upper chromatic number of a hypergraph, Australasian Journal of Combinatorics 11, 1995, 25-45.

[24] A. A. Zykov: Hypergraphs (in Russian), Uspekhi Mat. Nauk 29, 1974, 89-154. 